\title{
Der Nationalrat macht Nägel mit Köpfen
}

Yvonne Gilli, grüne Nationalrätin aus Wil/SG und praktizierende Komplementär-Ärztin, hat bei der Revision des Heilmittelgesetzes (HMG) eine Schlüsselrolle eingenommen. Sie konnte den Nationalrat überzeugen, die Zulassung von Komplementär- und Phytoarzneimitteln gegenüber dem Gesetzesentwurf des Bundesrats weiter zu vereinfachen. Wir haben mit Frau Gilli über die Behandlung des HMG im Nationalrat gesprochen.

Der Bundesrat hat im November 2012 die Botschaft erlassen. Wie beurteilen Sie den Vorschlag des Bundesrats bezüglich der Zulassung von Komplementärund Phytoarzneimitteln?

Dr. med. Yvonne Gilli: In der Botschaft des Bundesrats ist es ein klares Ziel, Komplementär- und Phytoarzneimittel erleichtert zuzulassen, weil dies Teil des Verfassungsauftrags ist. Diese klaren Aussagen der Regierung begrüsse ich sehr. Ich vermisse dann aber im Gesetzesentwurf detaillierte Vorschläge, wie das Ziel erreicht wird. Die Vorschläge des Bundesrats genügen leider nicht.

\section{Die parlamentarische Initiative von Marianne Kleiner aus dem Jahre 2007 wurde von beiden Kommissionen einstimmig angenommen. Wieso hat der Bundesrat die Forderungen nur teilweise übernommen?}

In der Kommissionsdebatte hat es sich gezeigt, dass vor allem Swissmedic die parlamentarische Initiative Kleiner nicht vollständig umsetzen will. Wir wurden immer wieder mit Aussagen konfrontiert, die für die Grosspharma zutreffen, die aber den spezifischen Bedingungen der Komplementär- und Phytohersteller nicht

gerecht werden, z.B. bezüglich der wirtschaftlichen Möglichkeiten. Die spezielle Situation der Homöopathie mit den individualisierten Kleinstmengen wurde kaum wahrgenommen, obwohl im HMG ein Sonderstatus sehr wichtig ist. Zentral erscheint mir also das fehlende Wissen über die spezifische Situation der Komplementärmedizin-Herstellung und teilweise die fehlende Bereitschaft, sich vertieft mit der Materie auseinanderzusetzen.

\begin{abstract}
Das HMG ist ein technisches, schwer verständliches Gesetz. Wissen die Kommissionsmitglieder, was es für die Zulassung bedeutet, z.B. auf klinische Studien oder toxikologische Prüfungen zu verzichten?
\end{abstract}

Nein, ein Grossteil der Kommissionsmitglieder weiss nicht im Detail, was die Folgen von Zulassungsvereinfachungen sind. HMG-Zulassungsfragen gehören nicht zum Allgemeinwissen. Es ist Spezialwissen, das man sich nur im Kontakt mit den Firmenvertretern aneignen kann. Der Schweizerische Verband für komplementärmedizinische Heilmittel (SVKH) hat uns fachlich sehr gut unterstützt. Bei technischen Fragen spielt die Verwaltung in der Kommission eine sehr wichtige Rolle. Einerseits müsste sie sich Praxiswissen der Komplementär-

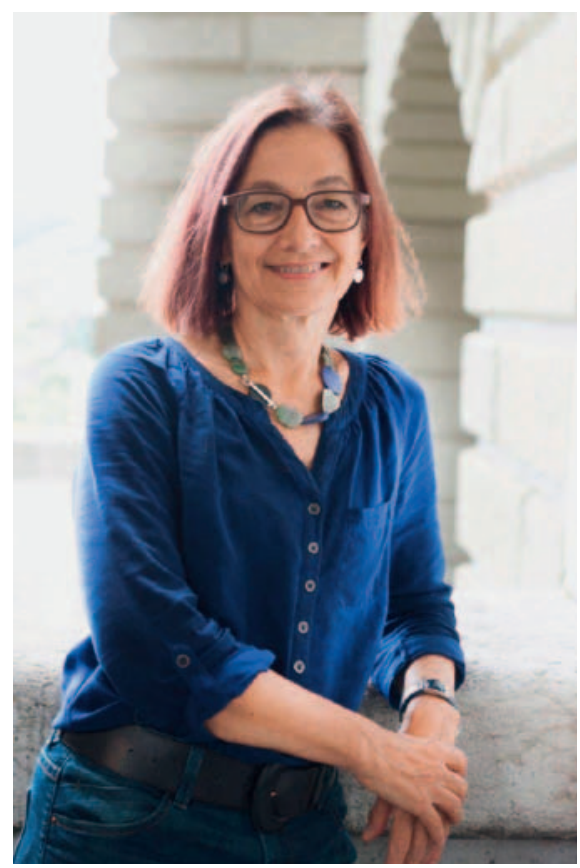

medizin-Produktion aneignen, andererseits müsste sie die Gesetzesvorlage neutral, korrekt und allgemein verständlich darlegen. Fehlt beides, dann macht sich jedes Kommissionsmitglied seinen eigenen Reim. Die Diskussion wird oft ideologisch und inkohärent geführt, wenn man die Folgen einer Gesetzesänderung nicht abschätzen kann. Es braucht immer ganz konkrete praktische Beispiele, sonst führen die Gegner ihr Standardargument aus, dass die Arzneimittelsicherheit des Patienten gefährdet sei.

\section{KARGER}

Fax +4976145207 14 
Dem Vernehmen nach leisteten die Behörden teilweise

Widerstand gegen Änderungsanträge. Wie konnten Sie die

Bedenken der Verwaltung in der Kommission entkräften?

Ich musste immer wieder frühzeitig das Expertenwissen bei den Arzneimittelherstellern des SVKH einholen, um mit Sachwissen argumentieren zu können. Neutrales Fachwissen ist schwer widerlegbar; oft musste es dann von der Verwaltung auch bestätigt werden. Ich musste die Änderungen mit meinen Kolleginnen und Kollegen in der Kommission vorbesprechen, damit sie sich mit ihren Bezugspersonen absprechen konnten und damit plausible Anliegen unterstützt wurden. Bei so technischen Fragen wie im HMG ist es aber oft schwierig zu erkennen, welche Folgen eine Änderung hat und welche Absichten dahinter stehen.

\section{Macht die Verwaltung eigentlich auch Politik und darf sie das?}

Die Verwaltung hat bei der HMGBeratung ausgesprochen viel Politik gemacht. Statt einer neutralen Information ist man oft einer Position gegenübergestanden. Weil der Gesundheitsminister bei der Beratung oft abwesend war, wusste man nicht, ob die Haltung mit dem Bundesrat abgesprochen war oder ob es sich um eine persönliche Meinung des Verwaltungsmitarbeitenden handelte. Das führte im Verlauf der Beratung auch zu einem gewissen Misstrauen. Man hatte nicht das Gefühl, dass die Verwaltung die Kommissionsmitglieder mit neutralem Hintergrundwissen informieren wollte. Alle Änderungsanträge wurden bekämpft, nicht nur diejenigen der Komplementärmedizin. Bei einer so umfassenden Gesetzesrevision braucht es aber eine gewisse Flexibilität seitens der Verwaltung. Änderungen einzugeben und zu besprechen ist ja die Aufgabe des Parlaments, sonst wäre die Beratung ja eine Farce.
Es sind über 100 Änderungsanträge eingegangen. Kann man die

Beratung überhaupt seriös durchführen?

Die Beratung einzelner Artikel dauerte manchmal sehr lange, gerade auch bei der Komplementärmedizin. Das wurde uns verschiedentlich vorgeworfen. Die Gesetzesrevision ist aber sehr umfassend. Es geht um ein Milliardengeschäft mit vielen Akteuren, von der Grosspharma über Komplementärhersteller bis hin zu Ärzten, Apothekern und Drogerien. Und natürlich nicht zu vergessen: Im Zentrum stehen eigentlich die Patientinnen und Patienten. Man musste darauf gefasst sein, dass viele Einzelanträge eingereicht werden, die wir alle behandeln müssen. Die Themen waren sehr unterschiedlich: Es braucht unterschiedliche Expertise bei der verein-

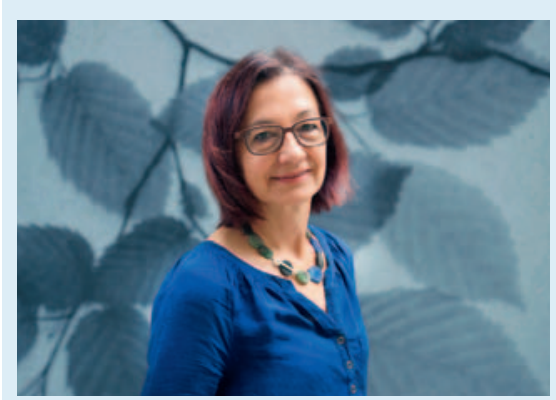

Dr. med. Yvonne Gilli ist Ärztin und Nationalrätin aus dem Kanton St. Gallen.

Nach der obligatorischen Schulzeit absolvierte Yvonne Gilli eine dreijährige Ausbildung als Pflegefachfrau. Auf dem zweiten Bildungsweg holte sie die Matura nach und studierte Medizin an der Universität Zürich. Sie ist Fachärztin für Allgemeinmedizin und hat FMH-Fähigkeitsausweise in Klassischer Homöopathie und Traditioneller Chinesischer Medizin. Seit 1996 arbeitet sie in ihrer eigenen Praxis mit den Schwerpunkten Gynäkologie und Komplementärmedizin in Wil/SG. Sie gehört dem Ärztenetzwerk xundart an.

Yvonne Gilli ist Mitglied der Grünen Partei. Sie sitzt in der Gesundheitskommission des Nationalrats und der Finanzkommission. Sie ist Mitglied der parlamentarischen Gruppe Komplementärmedizin und Co-Präsidentin der OdA Alternativmedizin. fachten Zulassung von Komplementärmedizin und bei strafrechtlichen Bestimmungen zu Korruption. Es war für ein einzelnes Kommissionsmitglied fast unmöglich, überall kompetent mitzudiskutieren.

\section{Die Zahl der zugelassenen Phytopräparate nimmt ab, Neuzulassungen gibt es kaum mehr. Wird mit dem revidierten HMG Innovation wieder möglich sein?}

Es ist unverständlich, dass Entwicklungen heute nicht mehr möglich sind, weil sie zu aufwendig und zu teuer sind. Grossmutters Pflanzenmittel wurden ja unreglementiert entwickelt. Es gibt eine riesige Zahl von neuen Pflanzen, die Heilpotenzial haben, aber ungenutzt sind. Sieht man, welche Möglichkeiten die Phytotherapie heute bereits mit relativ wenigen Pflanzen bietet, so kann man das Potenzial erahnen, das es noch gibt. Mit dem neuen HMG sollten vereinfachte Zulassungen möglich sein, allerdings bleibt es bei neuen Produkten schwierig. Der Markt ist klein, ein Gefahrenpotenzial bei gewissen Pflanzen sicherlich vorhanden. Die Sicherheitsbedenken dürfen aber nicht überwiegen. Oft fehlt der Behörde ein gewisses Augenmass. Voraussetzung ist auch, dass die Gesuche bei Swissmedic von Personen behandelt werden, die komplementärmedizinisches Know-how haben.

Innovation ist für mich zwingend, sonst sinkt der Arzneimittelschatz. Das erscheint mir sehr wichtig für die Zukunft der Heilmethoden zu sein.

\section{Der Nationalrat hat das Gesetz aus Sicht der Komplementär- und Phytohersteller beträchtlich verbessert. Welche Änderungen braucht es noch im Zweitrat?}

Die nationalen Vorgaben des HMG werden von den Kantonen sehr unterschiedlich vollzogen. Es gibt sehr 
strenge Kantonsbehörden, andere vernachlässigen die Vollzugsaufgaben bezüglich Arzneimitteln, Medizinprodukten und Nahrungsergänzungsmitteln weitgehend. Der Bund hat keine Möglichkeiten, Massnahmen vorzuschreiben oder einzugreifen, wenn die empfohlenen Massnahmen nicht umgesetzt werden. Bezüglich der Aufsicht und der Koordination des Vollzugs mit den Kantonen sollte der Bund mehr Kompetenzen erhalten. Er sollte die Vollzugsmassnahmen und die Informationstätigkeit koordinieren. Auch sollte er die Kantone verpflichten können, den Bund über Vollzugsmassnahmen sowie Kontrollund Untersuchungsergebnisse zu informieren. Das ist heute weitgehend eine Blackbox.

Die Lösung des Nationalrats für sogenannte Well-Established-Produkte ist ungenügend. Die Vorgabe, dass ein Produkt für eine vereinfachte Zulassung in fünf Ländern der Europäischen Union (EU) oder Europäischen Freihandelsassoziation (EFTA) seit mindestens 10 Jahren verwendet werden muss, ist eine zu hohe Hürde. Dieser Vorschlag basiert auf einer Hilfestellung der Verwaltung, die aber nicht praxistauglich ist.

Ein weiterer Punkt betrifft Fachpersonen, wie Komplementärtherapeuten oder Drogisten, die je nach Kanton nicht verschreibungspflichtige Arzneimittel abgeben dürfen. Da die Therapien der Komplementärmedizin stark individualisiert sind, fehlt oft gerade das Arzneimittel, das am geeignetsten wäre. Es wäre gut, einen neuen Artikel einzufügen. Dieser sollte den Fachpersonen erlauben, dass sie gewisse nicht zugelassene Produkte in ganz kleinen Mengen importieren dürfen, falls kein alternativ verwendbares Arzneimittel zugelassen ist.

Chancen für Komplementär- und Phytohersteller würden auch Vereinfachungen beim pädiatrischen Prüfkonzept bieten.
Welche wichtigsten Forderungen stellten die grossen Pharma-

Verbände im Rahmen der HMGRevision?

Die Verbände der Grosspharma wollten die Patente stärken bezüglich der Behandlung seltener Krankheiten und bezüglich der Heilmittel für Kinder. Beides sind Nischenmärkte. Der Forschungsaufwand ist bei beiden Kategorien hoch. Gerade bei seltenen Krankheiten braucht es neue Produkte für kleine Absatzmärkte.

\section{Welche Punkte waren der \\ Ärzteschaft wichtig?}

Für Ärzte sind mehrere Punkte relevant:

Welche Medikamente, die aktuell rezeptpflichtig sind, können in $\mathrm{Zu}$ kunft von Apothekern abgegeben werden. Es geht um eine Verschiebung des Absatzmarktes, weg von der Ärzteschaft hin zur Apothekerschaft.

Die zweite Frage ist, welche Verpflichtungen die Ärzte erfüllen müssen bezüglich der Verschreibung. Der Entwurf ging ja in der Gesundheitskommission so weit, dass für jedes Medikament ein Rezept ausgestellt werden muss, das abgegeben wird. Das hätte auch für rezeptfreie Medikamente gegolten; der Verwaltungsaufwand innerhalb der Arztpraxen wäre enorm gewesen und hätte Kosten von über 100 Millionen Franken verursacht.

Die dritte Frage ist der Umgang mit Rabatten beim Medikamenteneinkauf, die von ambulant oder stationär tätigen Leistungserbringern ausgehandelt werden. Es gibt immer noch die Forderung, dass Rabatte vollständig weitergegeben werden sollen. Die ist aus meiner Sicht nicht nachvollziehbar. Müssen Rabatte vollständig weitergegeben werden, dann gibt es keine Rabattverhandlungen mehr. Niemand wird mehr Zeit investieren, wenn man selber keinen Vorteil hat. Die Hersteller freuen sich dann, denn es muss der höchste Preis, nämlich der effektive Fabrikabgabepreis der Spezialitätenliste, bezahlt werden. Das ist nicht im Sinn der Leistungserbringer und nicht im Sinn der Patienten. Die Ärzte sind der Meinung, dass zusammen mit den Versicherern verhandelt werden soll, wie die Rabatte zu verwenden sind. Finanziert werden z.B. Qualitätszirkel in Ärztenetzwerken. Auch Versorgungsforschung wird mit diesen Rabatten finanziert. Gute Forschungsprojekte für die Patientenversorgung im ambulanten Bereich wären wohl nicht mehr möglich. Eine vollständige Rabattweitergabe wäre verheerend für Ärztenetzwerke, aber auch für Spitäler und Forschungsinstitutionen.

Zutreffend ist, dass teilweise $\mathrm{zu}$ hohe Rabatte verhandelt wurden und deren Verwendung für die Öffentlichkeit nicht immer transparent war. Das will man in Zukunft verhindern, indem im HMG eine Transparenzpflicht eingeführt wird. Rabatte müssen in den Büchern erfasst und gegenüber den Behörden unaufgefordert ausgewiesen werden. Die Rabatte können zwischen zwei starken Partnern, den Leistungserbringern und den Versicherern, ausgehandelt werden. Die Schwächen der heutigen Gesetzgebung wurden beseitigt. Die rechtlichen Grundlagen für den effektiven Vollzug wurden verbessert. Leider gibt es eine unheilige Allianz zwischen Mandatsträgern der Pharma, der Schweizerischen Volkspartei (SVP) und der Sozialdemokratischen Partei der Schweiz (SP). Das hat dazu geführt, dass eine solche vollständige Rabattweitergabe in der Kommission mehrheitsfähig war. Glücklicherweise hat der Nationalrat den Beschluss korrigiert.

Haben wir von SP und SVP das Referendum zu befürchten?

Das Referendum haben wir zu befürchten, wenn wesentliche Interessen der Grosspharma tangiert werden. Im 
- Komplementär- und neu auch Phytoarzneimittel werden generell vereinfacht zugelassen.

- Neue Kategorien werden geschaffen: «Well-Established Use», «Traditional Use», kantonale Heilmittel zur Überführung in eine nationale Zulassung.

- An kantonalen Zulassungen soll definitiv festgehalten werden.

- Im HMG werden erstmals die konkreten Zulassungsvereinfachungen pro Kategorie definiert.

- Bei Komplementärarzneimitteln mit Indikation und Phytoarzneimitteln können pharmakologische, toxikologische und klinische Prüfungen durch einen bibliographischen Nachweis von Wirksamkeit und Sicherheit oder Anwendungsbelege ersetzt werden.

Ende August 2014 begann die Beratung des HMG in der Gesundheitskommission des Ständerats. Es ist anzunehmen, dass der Ständerat gewisse Punkte ändert. Die Differenzen sind dann mit dem Nationalrat zu bereinigen. Nach dem Erlass des HMG werden die Verordnungen ausgearbeitet und in eine öffentliche Vernehmlassung geschickt. Mit der Inkraftsetzung wird frühestens im Herbst 2016 gerechnet.

Patentrecht wurden aber deren Maximalforderungen erfüllt. Auch wenn Referendumsdrohungen im Raum sind, glaube ich nicht, dass es ergriffen wird, weder von rechts noch von links. Die Ärzteschaft könnte allenfalls das Referendum ergreifen, wenn sie in allen wesentlichen Punkten zu den Verlierern gehört, wenn z.B. die Apotheker zu viele rezeptpflichtige Medikamente abgeben dürfen und wenn generell keine Rabatte mehr erlaubt sind. Insgesamt erachte ich ein Referendum als unwahrscheinlich.
Wie kann man sicherstellen, dass wir nicht nur ein gutes HMG haben, sondern auch Verordnungen gesetzeskonform erlassen werden?

Basierend auf den gemachten Erfahrungen bleibt dem Parlament nur eine Option: Man muss das Gesetz möglichst detailliert und gezielt revidieren, um auch im Bereich der Komplementärmedizin und der Phytoarzneimittel den Spielraum der Behörden einzuschränken. Mit dem geltenden
Gesetz wäre ja eine effektive vereinfachte Zulassung bereits möglich. Wir können aber real nachweisen, dass der pflanzliche Arzneimittelschatz verloren geht: Es gibt immer weniger zugelassene Phytopräparate.

\section{Der Bundesrat will die Preise in der Spezialitätenliste systema- tisch senken. Werden in Zukunft beim therapeutischen Querver- gleich pflanzliche Präparate mit chemischen Substanzen vergli- chen, dann verschwinden noch mehr sanfte Heilmittel vom Markt.}

Es braucht klar eine eigenständige Regelung für Komplementär- und Phytoarzneimittel. Sie dürfen nicht mit der Herstellung von synthetischen Heilmitteln verglichen werden. Preisverhandlungen sind zwar wichtig, aber generell muss nun Mass gehalten werden. Die Herstellungskosten, die Löhne und die Kaufkraft sind in der Schweiz höher; der internationale Vergleich hat Grenzen. Insofern habe ich für die Anliegen der Pharma-Hersteller auch grosses Verständnis. Gerade bei den Generika ist der Preis sensibel. Ist die Marge zu klein, dann kann in der Schweiz nicht mehr produziert werden. $\mathrm{Zu}$ tiefe Preise gefährden auch die Versorgungssicherheit.

Interview: Alexander Eitner 